\title{
Where fMRI and Electrophysiology Agree to Disagree: Corticothalamic and Striatal Activity Patterns in the WAG/Rij Rat
}

\author{
Asht Mangal Mishra, ${ }^{1,6}$ Damien J. Ellens, ${ }^{1}$ Ulrich Schridde, ${ }^{1}$ Joshua E. Motelow, ${ }^{1}$ Michael J. Purcaro, ${ }^{1}$ \\ Matthew N. DeSalvo, ${ }^{1}$ Miro Enev, ${ }^{1}$ Basavaraju G. Sanganahalli, ${ }^{4,6}$ Fahmeed Hyder,,${ }^{4,5,6}$ and Hal Blumenfeld ${ }^{1,2,3,6}$ \\ Departments of ${ }^{1}$ Neurology, ${ }^{2}$ Neurobiology, ${ }^{3}$ Neurosurgery, ${ }^{4}$ Diagnostic Radiology, ${ }^{5}$ Biomedical Engineering, and ${ }^{6}$ Core Center for Quantitative \\ Neuroscience with Magnetic Resonance, Yale University School of Medicine, New Haven, Connecticut 06520
}

The relationship between neuronal activity and hemodynamic changes plays a central role in functional neuroimaging. Under normal conditions and in neurological disorders such as epilepsy, it is commonly assumed that increased functional magnetic resonance imaging (fMRI) signals reflect increased neuronal activity and that fMRI decreases represent neuronal activity decreases. Recent work suggests that these assumptions usually hold true in the cerebral cortex. However, less is known about the basis of fMRI signals from subcortical structures such as the thalamus and basal ganglia. We used WAG/Rij rats (Wistar albino Glaxo rats of Rijswijk), an established animal model of human absence epilepsy, to perform fMRI studies with blood oxygen level-dependent and cerebral blood volume (CBV) contrasts at 9.4 tesla, as well as laser Doppler cerebral blood flow (CBF), local field potential (LFP), and multiunit activity (MUA) recordings. We found that, during spike-wave discharges, the somatosensory cortex and thalamus showed increased fMRI, CBV, CBF, LFP, and MUA signals. However, the caudate-putamen showed fMRI, CBV, and CBF decreases despite increases in LFP and MUA signals. Similarly, during normal whisker stimulation, the cortex and thalamus showed increases in CBF and MUA, whereas the caudateputamen showed decreased CBF with increased MUA. These findings suggest that neuroimaging-related signals and electrophysiology tend to agree in the cortex and thalamus but disagree in the caudate-putamen. These opposite changes in vascular and electrical activity indicate that caution should be applied when interpreting fMRI signals in both health and disease from the caudate-putamen, as well as possibly from other subcortical structures.

\section{Introduction}

Cortical-subcortical networks are integral to normal information processing in the brain but can also be exploited by abnormal paroxysmal activity, such as seizures. Absence seizures are brief staring spells, which occur most commonly in children and are accompanied by large-amplitude spike-and-wave discharges (SWDs) on electroencephalography (EEG). Neuroimaging, particularly functional magnetic resonance imaging (fMRI), has recently emerged as a powerful tool to elucidate the specific networks involved in SWD. However, fMRI signals are indirectly related to underlying neuronal activity, and paradoxical or unexpected relationships have been observed in brain disorders, in-

\footnotetext{
Received Dec. 22, 2010; revised July 27, 2011; accepted Aug. 14, 2011

Author contributions: A.M.M., U.S., B.G.S., F.H., and H.B. designed research; A.M.M., D.J.E., U.S., and B.G.S. performed research; A.M.M., D.J.E., U.S., J.E.M., M.J.P., M.N.D., and M.E. analyzed data; A.M.M. and H.B. wrote the paper.

This work was supported by NIH Grants R01 NS049307 (H.B.), R01 MH067528 (F.H.), and P30 NS052519 (F.H.), Epilepsy Foundation Award 123505 (A.M.M.), and the Betsy and Jonathan Blattmachr family. We thank Dr. Bei Wang and Xiaoxian Ma for technical assistance with animal preparation, Nathan Danielson for help with software development, Dario Englot for helpful comments on this manuscript, and the engineering staff of the Magnetic Resonance Research Center (http://mrrc.yale.edu) and Quantitative Neuroscience with Magnetic Resonance (http:// qnmr.yale.edu) for hardware maintenance and radio frequency probe construction.

Correspondence should be addressed to Dr. Hal Blumenfeld, Yale Departments of Neurology, Neurobiology, and Neurosurgery, 333 Cedar Street, New Haven, CT 06520-8018. E-mail: hal.blumenfeld@yale.edu.

DOI:10.1523/JNEUROSCI.0101-11.2011

Copyright $\odot 2011$ the authors $\quad 0270-6474 / 11 / 3115053-12 \$ 15.00 / 0$
}

cluding epilepsy (D’Esposito et al., 2003; Schridde et al., 2008; Shih et al., 2009). In the cerebral cortex, previous work suggests that fMRI and neuronal activity agree both under normal conditions (Logothetis et al., 2001; Smith et al., 2002; Shmuel et al., 2006) and during SWD (Nersesyan et al., 2004a,b). However, subcortical structures have not been thoroughly investigated. We therefore sought, through use of a rat SWD model, to relate fMRI signals in cortical and subcortical structures to direct electrical recordings of neuronal activity in the same networks.

Human fMRI studies of SWD have shown fMRI increases and decreases in the cortex, mainly increases in the thalamus and decreases in the basal ganglia (Archer et al., 2003; Gotman et al., 2005; Hamandi et al., 2006; Moeller et al., 2008a; Bai et al., 2010; Berman et al., 2010). Blood oxygenation level-dependent (BOLD) fMRI signal depends on cerebral blood flow (CBF), cerebral blood volume (CBV), and the cerebral metabolic rate of oxygen consumption (Ogawa et al., 1993, 1998). However, relationships between hemodynamic and neuronal activity changes in cortical-subcortical structures during SWD are not known.

We therefore sought to study the relationship between fMRI signals and underlying neuronal activity in the Wistar albino Glaxo rat of Rijswijk (WAG/Rij), an established animal model of human absence epilepsy (Coenen et al., 1992; Coenen and Van Luijtelaar, 2003). Previous electrophysiological and fMRI studies of SWD in WAG/Rij rats have shown intense involvement of a 
specific region of the face cortex overlapping somatosensory barrel cortex (S1BF) (Meeren et al., 2002; Nersesyan et al., 2004b). In addition, previous work has demonstrated subcortical network involvement of the thalamus and basal ganglia during SWD in WAG/Rij rats (Lasoń et al., 1992; Nersesyan et al., 2004a) and related models (Deransart et al., 1998; Slaght et al., 2004; David et al., 2008).

We performed simultaneous EEG-fMRI to measure BOLDand CBV-related changes in cortical and subcortical networks during SWD. With this approach, we identified prominent increases in somatosensory barrel cortex and thalamus and decreases in the basal ganglia. We next investigated the physiological basis of these changes through multiunit, local field potential (LFP), and laser Doppler flowmetry CBF measurements in these three regions, comparing similar experiments in SWD and normal whisker stimulation. $\mathrm{PMRI}$ increases in cortex and thalamus during SWD were associated with the expected increases in electrophysiological activity and CBF. However, the basal ganglia showed paradoxical fMRI and CBF decreases despite increases in neuronal electrical activity.

\section{Materials and Methods}

All experimental procedures were in full compliance with Yale University Institutional Animal Care and Use Committee protocols approved in agreement with the National Institutes of Health. Data from 43 adult (36 female) WAG/Rij rats (Harlan) aged $10 \pm 1$ months (mean \pm SEM) with an average weight of $220 \pm 1 \mathrm{~g}$ were included in this study. Twenty-two rats were used for the fMRI experiments, and 21 rats were used for combined neurophysiological and laser Doppler flowmetry CBF recordings.

\section{Animal preparation and general procedures}

Anesthesia was induced using 1.5\% halothane (Halocarbon Products) and later switched to intravenous fentanyl ( $40 \mu \mathrm{g} / \mathrm{kg}$; Hospira) and intraperitoneal haloperidol (1 mg/kg; Bedford Labs) because fentanylhaloperidol anesthesia does not suppress SWDs (Pinault et al., 1998). To prevent any movements during the fMRI experiments and to facilitate artificial breathing, rats were paralyzed by intravenous injections of 0.5 $\mathrm{mg} / \mathrm{kg}$ D-tubocurarine chloride (Sigma-Aldrich) every $2 \mathrm{~h}$, tracheotomized, and artificially ventilated as in previous investigations (Nersesyan et al., 2004a; Englot et al., 2008). Level of anesthesia was assessed by heart rate, blood pressure, and continuous EEG monitoring. Anesthesia was maintained by injection of a similar dose of fentanyl and haloperidol every $45 \mathrm{~min}$. One femoral vein was cannulated, and an intraperitoneal line was placed (Intramedic PE10 tubing; BD Biosciences) for the injections of anesthetics. A femoral artery was cannulated (Intramedic PE50 tubing; BD Biosciences) for continuous arterial blood pressure monitoring and periodic blood sampling for measurements of blood gases and pH using either an i-STAT 1 (i-STAT Corporation) or an ABL 5 blood gas analyzer (Radiometer Analytical). All physiological values (blood gases, mean arterial blood pressure, and $\mathrm{pH}$ ) were maintained within physiological range throughout the experiments as described previously (Englot et al., 2008; Schridde et al., 2008), by adjusting respiration and level of anesthesia throughout the experiments. The body temperature of the rat was monitored with a rectal probe and kept constant at $37^{\circ} \mathrm{C}$ using a water heating pad (model \#TP3E; Gaymar Industries). MRI experiments and neurophysiological recordings were acquired over a time period of 20-30 min. Under these conditions, animals typically have multiple episodes of spontaneous SWDs as described previously (Nersesyan et al., 2004a,b).

\section{MRI experiments}

Twenty-two rats were used for fMRI experiments, obtained in a total of $51 \mathrm{fMRI}$ experimental runs. BOLD data were acquired from all 22 rats, and five of these rats were also used for CBV measurements after at least one successful BOLD run. Experiments with no seizures, continuous seizures resulting in lack of baseline EEG, poor systemic physiology, motion in $\mathrm{fMRI}$ images, or abortion of fMRI data acquisition attributable to technical reasons were excluded from analysis. BOLD fMRI experiments were performed without exogenous contrast administration. For CBV-weighted fMRI experiments, a dose of $10-18 \mathrm{mg} / \mathrm{kg}$ iron oxide contrast agent [Combidex (ferumoxtran-10); Advanced Magnetics] was administered intravenously $10-15$ min before image acquisition.

During MRI recordings, the rat was positioned prone in a specially designed plastic holder with the head fixed and bregma positioned at the center of the surface coil. The animals were then inserted into the magnet with its head positioned at the isocenter of the magnet. EEG signals were acquired simultaneously with fMRI using a pair of 1-mm-diameter carbon-filament electrodes (WPI). These carbon filaments were placed between the scalp and the upper surface of the skull in the frontal and occipital areas, with the exposed portion of each electrode running in the coronal plane crossing the midline (from left to right), and secured to the skin with tissue glue (3M Vetbond; 3M Animal Care Products) to minimize MRI signal distortion (Englot et al., 2008). The EEG signals were acquired in differential mode between the two carbon-filament electrodes, amplified $(100 \times)$ and filtered $(1-30 \mathrm{~Hz})$ using a model 79D Data Recording System (Grass Instruments). EEG signals were digitized and recorded (sampling rate, $1000 \mathrm{~Hz}$ ) using a CED Micro 1401 and Spike 2 software (Cambridge Electronics Design).

All fMRI experiments were acquired on a 9.4 Tesla Bruker (Bruker Avance) horizontal bore (16 cm internal diameter) spectrometer, equipped with passively shielded shim/gradient coils $(47.5 \mathrm{~g} / \mathrm{cm})$ operating at $400.5 \mathrm{MHz}$ for protons. The transceiver system consisted of a surface coil (15.18 mm diameter) for transmission of radio frequency pulses and receiving. To optimize the homogeneity of the static magnetic field, the system was shimmed before each experiment using global manual shimming.

Anatomical images for each animal were acquired with 5 or 11 interlaced slices in the coronal plane using the fast low-angle shot sequence with the following parameters: repetition time (TR), $500 \mathrm{~ms}$; echo time (TE), $6 \mathrm{~ms}$; flip angle, $40-55^{\circ}$; field of view (FOV), $25 \times 25 \mathrm{~mm}$; $256 \times$ 256 matrix size; in-plane resolution, $98 \times 98 \mu \mathrm{m}$; and slice thickness, $1000 \mu \mathrm{m}$, no gap. BOLD and CBV-weighted fMRI data were obtained in the same planes as anatomical images. We used single-shot spin echo, echo planar imaging (SE-EPI; 22 experimental runs in 16 animals) or echo planar imaging, gradient echo (GE-EPI; 25 experimental runs in 13 animals; seven animals had both SE and GE acquisitions) and obtained similar results with both approaches. SE-EPI data were acquired with the following parameters: TR, $1000 \mathrm{~ms}$; TE, $25 \mathrm{~ms}$; excitation flip angle, $90^{\circ}$; inversion flip angle, $180^{\circ}$; FOV, $25 \times 25 \mathrm{~mm}$; $64 \times 64$ matrix size; in-plane resolution of $390 \times 390 \mu \mathrm{m}$; and slice thickness, $1000 \mu \mathrm{m}$. All SE-EPI experiments were acquired with 11 slices. The 11 slices were acquired over $1000 \mathrm{~ms}$, followed by a 2 or $5 \mathrm{~s}$ pause before the next image onset so that EEG could readily be interpreted during data acquisition; time between onset of consecutive image acquisitions was therefore 3 or $6 \mathrm{~s}$. We acquired 300-600 images per run, resulting in a total imaging time of $1800 \mathrm{~s}$ for most experimental runs (a shorter imaging time of 900 or $1500 \mathrm{~s}$ was used for two runs). GE-EPI data were acquired with following parameters: TR, $1000 \mathrm{~ms}$; TE, $13 \mathrm{~ms}$; FOV, $25 \times 25 \mathrm{~mm}$; $64 \times 64$ matrix size; and in-plane resolution of $390 \times 390 \mu \mathrm{m}$. For most GE-EPI runs, slice thickness was $2000 \mu \mathrm{m}$, and five slices were acquired over 1000 $\mathrm{ms}$, followed by a $2 \mathrm{~s}$ pause; time between consecutive image onsets was therefore $3 \mathrm{~s}$. We acquired 300-400 images per run, with a total imaging time of $900 \mathrm{~s}$ for most runs. For two GE-EPI runs, slice thickness was 1000 $\mu \mathrm{m}$ (like in SE-EPI runs), resulting in 11 slices acquired every $3 \mathrm{~s}$, with 400 total images obtained over $1200 \mathrm{~s}$. Images from the first $48 \mathrm{~s}$ of each run were discarded from analysis.

\section{Neurophysiological and laser Doppler flowmetry CBF recording experiments}

Twenty-one rats were used for acute electrophysiological and laser Doppler flowmetry CBF recordings, acquired in 34 experimental runs, each lasting $\sim 30 \mathrm{~min}$. Experiments with no seizures, poor systemic physiology, or lack of histological verification of probe placements were excluded from analysis. In each experiment, signals were recorded simultaneously either from $\mathrm{S} 1 \mathrm{BF}$ and caudate-putamen $(\mathrm{CPu})$ or from 

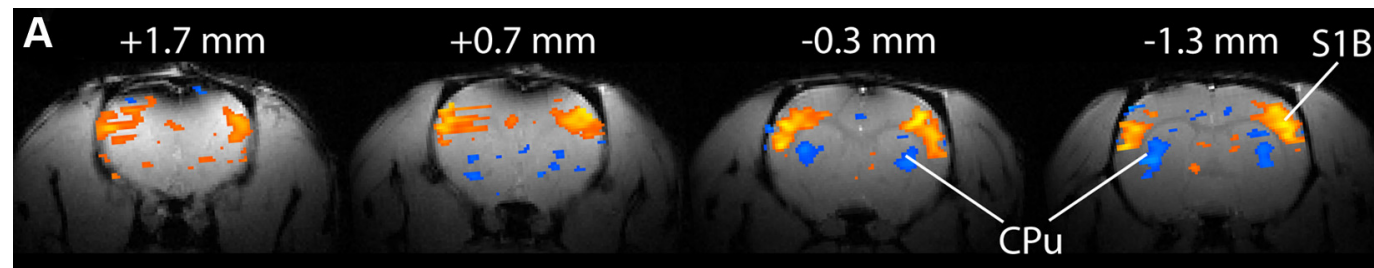

$-2.3 \mathrm{~mm}$

$-3.3 \mathrm{~mm}$

$-4.3 \mathrm{~mm}$

$-5.3 \mathrm{~mm}$
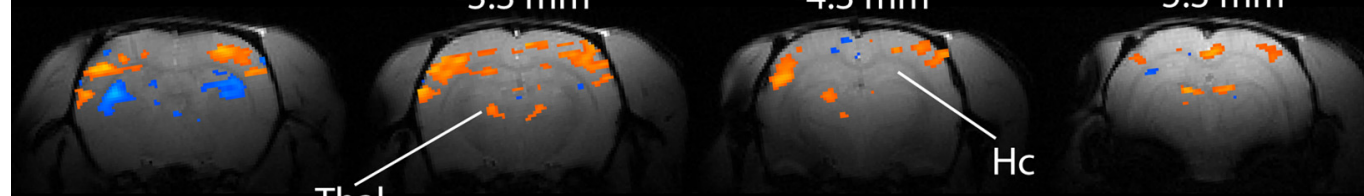

Thál
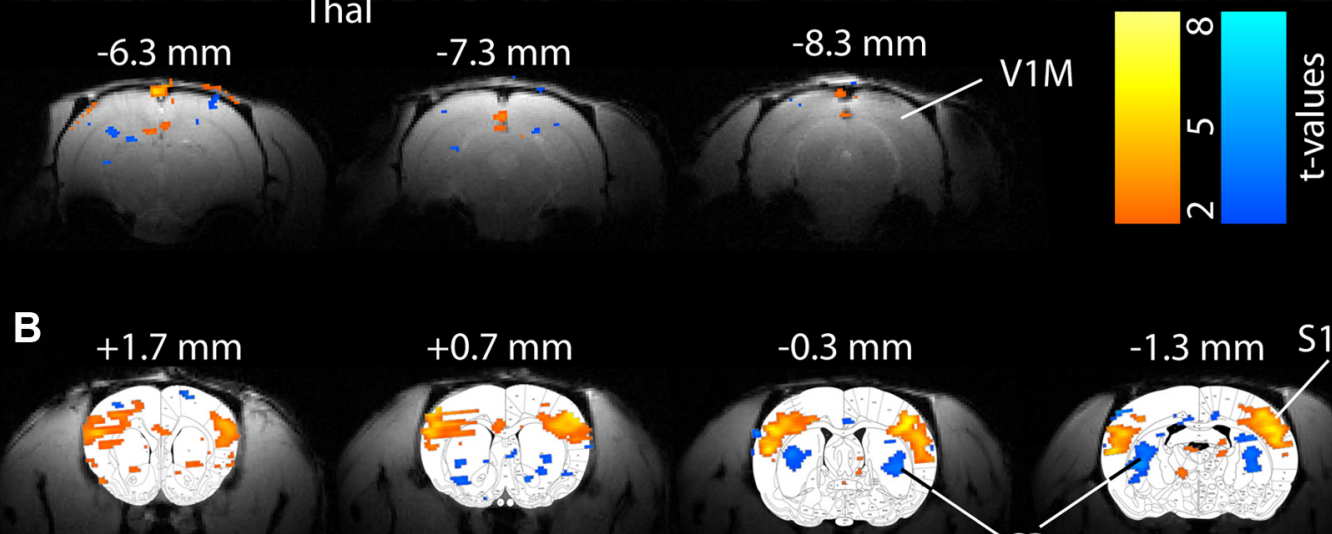

$-0.3 \mathrm{~mm}$

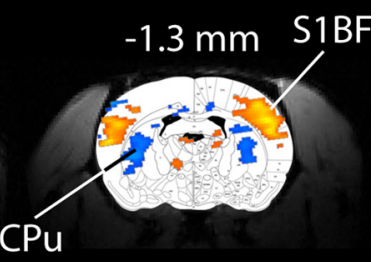

$-2.3 \mathrm{~mm}$

$-3.3 \mathrm{~mm}$

$\mathrm{CPu}$
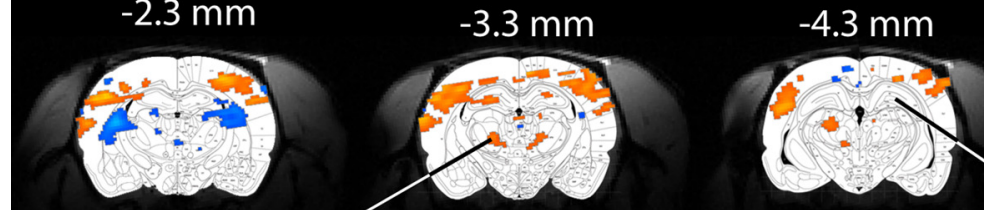

$\mathrm{Hc}$

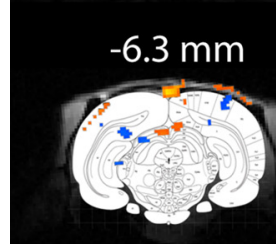

Thal
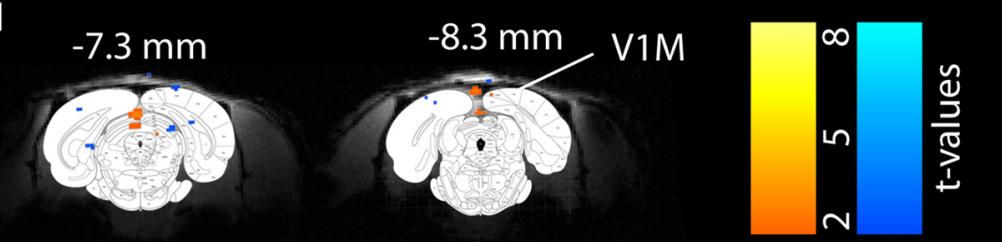

Figure 1. Example of BOLD fMRI changes $2-4 \mathrm{~s}$ after SWD onset in a WAG/Rij rat at $9.4 \mathrm{~T}$ and overlay of $t$ maps on rat brain atlas. $A, S 1 B F$ and thalamus (Thal) show prominent increases in BOLD signal during SWDs. Prominent BOLD decreases are present in the (Pu. No changes are seen in V1M or hippocampus (Hc). Smaller changes are seen in other areas. Simultaneous EEG acquired during fMRI was used to identify images obtained $2-4 \mathrm{~s}$ after SWD onset for comparison with baseline images obtained immediately before start of SWDs. Results are displayed as $t$ maps of BOLD fMRI signal superimposed on high-resolution anatomical images. $t$ values were generated using a paired $t$ test in which pairs consisted of one seizure acquisition taken $2-4 \mathrm{~s}$ after seizure onset and the baseline images acquired just preceding SWD onset ( $n=26$ SWD episodes). Slices are shown from anterior to posterior, with approximate coordinates relative to bregma (Paxinos and Watson, 1998). Color bars indicate $t$ values for increases (warm colors) and decreases (cold colors). Threshold value $t>2 . B, 0$ verlay of $t$ maps on rat brain atlas (Paxinos and Watson, 1998, reproduced with permission) shows anatomical locations of BOLD fMRI signal increases and decreases.

S1BF and ventral posterior medial nucleus of thalamus (VPM) during multiple SWD.

Rats were mounted in a stereotaxic frame (David Kopf Instruments) in a Faraday cage, the skin and underlying tissue over the skull were removed, and small burr holes were drilled. For simultaneous electrophysiology and local CBF measurements, we used a high-impedance 2-4 M $\Omega$ tungsten microelectrode (part \#UEWMGGSEDNNM; FHC) attached to an Oxyflo MNP 110 XP needle laser Doppler flow probe (outer diameter, $0.5 \mathrm{~mm}$ ), connected to an Oxyflo model 4000 (Oxford Optronix). For each experiment, a microelectrode/laser Doppler probe assembly was placed in two of the following three brain region at a time: S1BF [anteroposterior (AP), $-2.3 \mathrm{~mm}$; mediolateral (ML), $-5.02 \mathrm{~mm}$ ], VPM (AP, $-3.3 \mathrm{~mm}$; ML, $+3.20 \mathrm{~mm}$ ), and caudate-putamen (CPu) (AP, $0.0 \mathrm{~mm}$; $\mathrm{ML},+4.00 \mathrm{~mm})$, measured from bregma. All coordinates were determined according to a standard rat brain atlas (Paxinos and Watson,
1998) and corresponded to maximum fMRI signal changes (Fig. 1 A,B). We matched coordinates as closely as possible; however, these coordinates may be off by $\pm 1 \mathrm{~mm}$ in MRI slices compared with rat brain atlas (Paxinos and Watson, 1998). Duratomy was performed to facilitate microelectrode/probe insertion, and the recording probes were lowered under auditory and visual guidance until touching the cortex, which marked the SI zero point for microelectrode/probe insertion. The probes were then lowered slowly in CPu and VPM perpendicular to skull and at $30^{\circ}$ angle to skull in S1BF to a final SI depth of 3.5-4.5 mm for $\mathrm{CPu}$, $0.6-1.6 \mathrm{~mm}$ for $\mathrm{S} 1 \mathrm{BF}$, and $4.5-5.5 \mathrm{~mm}$ for VPM. The tip of the microelectrode was placed $\sim 200-400 \mu \mathrm{m}$ below the laser Doppler probe to ensure that the sampling volumes of all measurements were overlapping. Signals from the microelectrodes were first acquired broadband (1 Hz to $10 \mathrm{kHz}$ ) and amplified using a microelectrode alternating current amplifier model 1800 (A-M Systems) with $100 \times$ gain during spontaneous 
SWD in WAG/Rij rats. Signals were then filtered with a model 3363 Butterworth filter ( $24 \mathrm{~dB} /$ octave attenuation) (Krohn-Hite) into lowfrequency band for LFP $(1-100 \mathrm{~Hz})$ and high-frequency band for multiunit activity (MUA) (400-10 kHz) (Shmuel et al., 2006; Englot et al., 2008; Schridde et al., 2008). All signals were recorded using Spike 2 software and digitized with a Power 1401 (Cambridge Electronics Design) at a sampling rate of $1 \mathrm{kHz}$ for EEG, LFP, and CBF and $20 \mathrm{kHz}$ for MUA.

Whisker stimulation was performed with electrophysiological and CBF recordings in the same animals in which SWDs were measured using methods described previously (Nersesyan et al., 2004b). We performed mechanical stimulation of all whiskers in harmony on the rat's left or right snout, depending on recording side, with a wooden probe using forward and backward movements without touching the skin at $\sim 10 \mathrm{~min}$ intervals, with stimulus duration of $4 \mathrm{~s}$ and at a frequency of $5 \mathrm{~Hz}$. Electrophysiological and CBF recordings were performed from S1BF, $\mathrm{VPM}$, and $\mathrm{CPu}$ to measure the response to contralateral whisker stimulation.

\section{Histological verification of recording sites}

At the end of experiments, rats were killed with an intraperitoneal injection of Euthasol (Virbac), brains were harvested, and all microelectrode and laser Doppler probe assembly placements were verified histologically. The brains were rapidly removed and transferred to $4 \%$ phosphatebuffered paraformaldehyde, $\mathrm{pH}$ 7.4. After 1 week of fixation in $4 \%$ paraformaldehyde in PBS, brains were then immersed in 30\% sucrose for at least $2 \mathrm{~d}$ until they sank in sucrose solution. All brains $(n=21)$ were sliced to $60 \mu \mathrm{m}$ thick slices, transferred on gelatin-coated glass slides, and stained with cresyl violet, and images were taken to ensure correct position of recording probes in the brain. Locations of the microelectrode/ laser Doppler probe assembly were marked based on tissue damage seen on cresyl violet-stained histology sections, and recorded sites were mapped on coronal brain section schematics from a standard atlas (see Fig. 4) (Paxinos and Watson 1998). All nonconfirmed experiments were discarded from the analysis.

\section{EEG analysis and SWD definition}

All EEG signals acquired during fMRI experiments were first processed using Spike 2 software, and methods were used to reduce magnetic fieldinduced artifacts similar to those described previously (Nersesyan et al., 2004a; Englot et al., 2008; Schridde et al., 2008). All EEG data acquired during fMRI and neurophysiology experiments were marked for individual SWD onset and offset time in Spike 2 software manually using a script provided by Cambridge Electronics Design. SWDs were defined as largeamplitude (more than two times the peak-to-peak amplitude baseline EEG) rhythmic 5-8 Hz discharges with typical spike-wave morphology lasting $>1.0 \mathrm{~s}$ (Coenen and Van Luijtelaar, 1987; Blumenfeld et al., 2008). The SWD onset and end time marks were used for all subsequent analyses of fMRI and physiology data. Overall mean SWD duration for all experiments was $2.96 \pm 2.06 \mathrm{~s}$ (mean $\pm \mathrm{SD} ; n=2760$ SWDs total), similar to that in previous work (Nersesyan et al., 2004a,b).

To facilitate comparison of fMRI and physiology data, the onset time of each SWD was used as "time 0 " for binning and analyzing time series data across multiple SWDs. Temporally aligning the onset time of multiple SWDs allowed mean changes to be determined in specific time bins before and after SWD onset.

\section{fMRI data analysis and statistics}

fMRI images were processed using an in-house program running on a MATLAB platform (MathWorks) written by the authors (F.H., J.E.M., and M.J.P.). Although rats were paralyzed during experiments, all fMRI series were first screened for movement artifacts using a movie function and center-of-mass analysis, restricted to voxels within the brain boundaries, to ensure that all runs exhibited movement of $<20 \%$ of a pixel in either the $x$ or $y$ direction as described previously (Nersesyan et al., 2004a; Schridde et al., 2008; DeSalvo et al., 2010).

Analysis of all fMRI data was performed using $2 \mathrm{~s}$ time bins, temporally aligning all SWD episodes by setting SWD onset as time 0 . Baseline was obtained for each SWD episode using any images acquired during the $6.5 \mathrm{~s}$ interval preceding each $\mathrm{SWD}$, with the additional requirement that no images were included in baseline if a preceding SWD event continued or ended within $3 \mathrm{~s}$ of image onset. If more than one image occurred during the baseline interval for any given SWD, these were averaged to obtain a single baseline image. For electrophysiology (higher sampling rate), we used $2 \mathrm{~s}$ baselines because this provided enough baseline signal for comparison, but results were not altered if a longer (e.g., $6.5 \mathrm{~s}$ ) baseline was used (data not shown). All fMRI time series contained a spatially inhomogeneous component attributable to MR scanner drift over the 30 min experimental run (Smith et al., 1999; Foerster et al., 2005; Benner et al., 2006). This component was removed by subtracting a third-order polynomial fit of each dataset. We verified that seizure events did not contribute to polynomial fit because seizures are very brief $(\sim 3 \mathrm{~s})$ events compared with $30 \mathrm{~min}$ of fMRI run, and seizures were on average distributed evenly throughout the scan time (data not shown).

$B O L D$ t map calculations. BOLD fMRI $t$ maps were calculated using a paired $t$ test in which each pair consisted of one baseline image (defined above) and one seizure image acquired in the time bin 2-4 s after seizure onset, because this time bin showed maximum changes in fMRI and electrical signals. $t$ maps were obtained across multiple SWD episodes from each experimental run and displayed using threshold $t>2$. The $t$ maps were superimposed onto corresponding high-resolution anatomical images from the same animal, and regional changes were identified based on a standard rat atlas (Paxinos and Watson, 1998).

$C B V$ t map calculations. For CBV analysis, additional calculations were necessary. The fMRI signal obtained after iron oxide contrast administration is a combination of the BOLD signal attributable to deoxyhemoglobin and of the CBV-related signal attributable to the exogenous contrast. With large CBV changes, the contribution of the BOLD signal is relatively small, and therefore $\mathrm{CBV}$ changes can be measured to a first approximation as the change in total fMRI signal in the presence of iron oxide contrast. This approach was used in a recent chemically induced tonic-clonic seizure model (Schridde et al., 2008), although a much higher contrast dose was needed in a rat model of smaller partial seizures (Englot et al., 2008). This approach, however, is not a reliable estimate of CBV changes during SWD because neuronal activity and CBF changes are relatively small (Nersesyan et al., 2004a,b). If during a given perturbation there is an obligatory BOLD signal increase (attributable to endogenous hemoglobin as the contrast agent), at the same time during a CBV experiment, there is a necessary CBV signal decrease (attributable to exogenous superparamagnetic nanoparticles as the contrast agent). Because both signal changes are attributable to changes in the transverse relaxation rate of tissue water, the BOLD effect has to be removed from the CBV effect for the volume change to be quantified during SWD, as shown by the following equation (Kennan et al., 1998; Hyder, 2004):

$$
\frac{\Delta \mathrm{CBV}}{\mathrm{CBV}}=\frac{\ln \left(1+\frac{\Delta S_{\mathrm{CBV}}}{S_{\mathrm{CBV}}}\right)-\ln \left(1+\frac{\Delta S_{\mathrm{BOLD}}}{S_{\mathrm{BOLD}}}\right)}{\ln \left(\frac{S_{\mathrm{CBV}}}{S_{\mathrm{CBV}}^{0}}\right)-\ln \left(\frac{S_{\mathrm{BOLD}}}{S_{\mathrm{BOLD}}^{0}}\right)},
$$

where $\triangle \mathrm{CBV} / \mathrm{CBV}$ is the calculated fractional change in $\mathrm{CBV}$ during an individual SWD, $\Delta S_{\mathrm{CBV}}$ is fMRI signal changes during individual SWDs with contrast, $S_{\mathrm{CBV}}$ is baseline fMRI signal with contrast for individual SWD, $\Delta S_{\text {BOLD }}$ is mean fMRI signal changes across SWD without contrast, and $S_{\text {BOLD }}$ is mean baseline fMRI signal across SWD without contrast. For these calculations, $S_{\mathrm{CBV}}$ data were obtained during multiple SWD episodes with contrast, and mean $S_{\mathrm{BOLD}}$ data were obtained from another run (without contrast) from the same animal.

$S_{\mathrm{CBV}}^{0}$ and $S_{\mathrm{BOLD}}^{0}$ were defined as the MRI signal as TE approaches 0 , with and without contrast, respectively (Hyder, 2004). We obtained these values for both GE and SE sequences by acquiring structural images with TE ranging from 31 to $5 \mathrm{~ms}$ and computing mean values of absolute signal intensity across slices. These mean values were plotted, and a thirdorder polynomial regression was used to estimate $S_{\mathrm{BOLD}}^{0}$ and $S_{\mathrm{CBV}}^{0}$ as TE approaches 0 .

As with BOLD $t$ map calculations, we evaluated CBV $t$ maps in the 2-4 $\mathrm{s}$ time bin after SWD onset, because this represents peak changes. Using the above equation, we calculated $\Delta \mathrm{CBV} / \mathrm{CBV}$ using $\Delta S_{\mathrm{CBV}} / S_{\mathrm{CBV}}$ in the $2-4 \mathrm{~s}$ bin for each SWD together with the mean value for $\Delta S_{\mathrm{BOLD}} / S_{\mathrm{BOLD}}$ 
Table 1. Summary of anatomical brain areas involved in FMRI BOLD and CBV increases and decreases across animals

\begin{tabular}{|c|c|c|c|c|c|c|}
\hline & S1BF & Thalamus & $\begin{array}{l}\text { Anterior } \\
\text { cingulate } \\
\text { cortex }\end{array}$ & $\begin{array}{l}\text { Posterior cingulate/ } \\
\text { retrosplenial cortex }\end{array}$ & $\begin{array}{l}\text { Superior } \\
\text { colliculi }\end{array}$ & $\begin{array}{l}\text { Caudate- } \\
\text { putamen }\end{array}$ \\
\hline BOLD increases & $22(45)$ & $22(39)$ & $20(31)$ & $19(33)$ & $9(13)$ & $0(0)$ \\
\hline BOLD decreases & $0(0)$ & $0(0)$ & $2(2)$ & $1(1)$ & $0(0)$ & $18(30)$ \\
\hline CBV increases & $5(6)$ & $5(6)$ & $4(5)$ & $3(3)$ & $0(0)$ & $0(0)$ \\
\hline CBV decreases & $0(0)$ & $0(0)$ & $1(1)$ & $1(1)$ & $0(0)$ & $5(6)$ \\
\hline
\end{tabular}

Data represent total number of animals (number of experimental runs) showing increases or decreases on review of $t$ maps for each experiment. A total of 22 animals ( 45 experimental runs) were analyzed for BOLD, and five animals (six experimental runs) were analyzed for CBV.

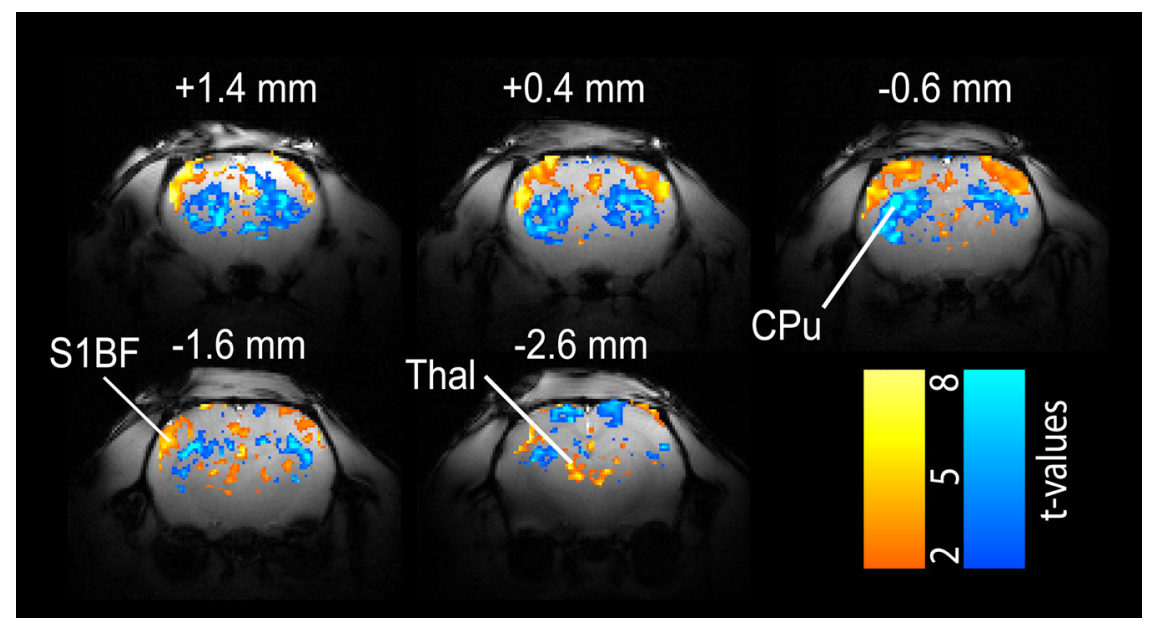

Figure 2. Example of CBV changes during SWDs in a WAG/Rij rat at 9.4 T. S1BF and thalamus (Thal) show prominent increases in CBV signal during SWD, whereas (Pu shows decreases, with similar regions and direction of changes compared with BOLD fMRI (Fig. 1). Simultaneous EEG acquired during fMRI was used to identify images obtained 2-4 s after SWD onset. $t$ maps of CBV changes during SWD were calculated as described in Materials and Methods and superimposed on anatomical images. Slices are shown from anterior to posterior, with approximate coordinates relative to bregma (Paxinos and Watson, 1998). Color bars indicate $t$ values for increases (warm colors) and decreases (cold colors). $n=6$ SWDs during CBV and 10 SWDs during BOLD in the same animal used for (BV calculations (see Materials and Methods). Threshold value $t>2$.

\section{A}
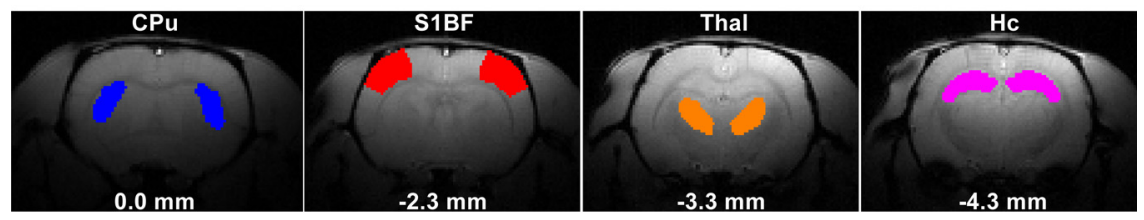

B

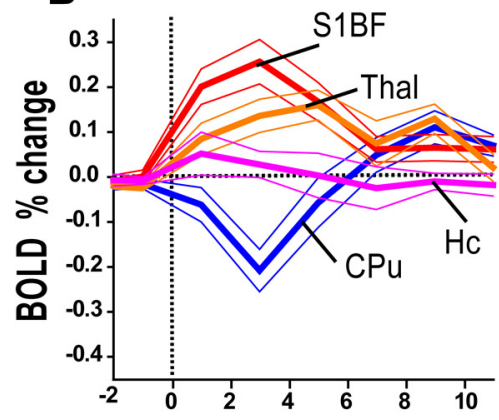

Time (s)

Figure 3. BOLD fMRI and CBV increase in S1BF and thalamus (Thal) but decrease in CPu during SWDs. Time course of signals are displayed as percentage change relative to pre-seizure baseline data (see Materials and Methods for definition of baseline). $\boldsymbol{A}$, ROl examples displayed on structural images for a single animal, based on anatomical regions (Paxinos and Watson, 1998). Similar ROls were created for all animals. $\boldsymbol{B}$, BOLD signals changes ( $n=22$ animals; data are from 1856 SWDs total). C, CBV signal changes ( $n=5$ animals; data are from 418 SWD total). Time courses are displayed as mean \pm SEM, with 2 stime bins. Vertical line at time $=0$ marks SWD onset. Hc, Hippocampus. in the 2-4 s time bin from another run in the same animal without contrast. We then used a one-sample $t$ test to determine the significance of $\triangle \mathrm{CBV} / \mathrm{CBV}$ across multiple SWD episodes from each experimental son, 1998).

and $C B V$ time courses. Time course analysis focused on specific ost consistently, namely S1BF, thalamus, and CPu (Fig. 1B) (see Fig. (s) Fig. 4). ROI time course analyses were done using an in-house program running on a MATLAB platform. ROIs were drawn separately for each animal in the coronal plane of high-resolution anatomical images (see Fig. $3 A$ ) guided by a rat brain atlas (Paxinos and Watson, 1998). Baseline images and images for $2 \mathrm{~s}$ time bins were obtained as described above. The mean signal was calculated for each ROI at each point in time. The percentage change relative to baseline was then calculated from $2 \mathrm{~s}$ before to $11 \mathrm{~s}$ after each SWD. Data were pooled by averaging across SWD within the experimental run and then across runs for each animal to yield a single time course in $2 \mathrm{~s}$ time bins per animal. Mean time courses and statistics were then calculated across animals. To compare amplitude of changes in different regions from fMRI and physiology measurements, we analyzed the mean change in the 2-4 $s$ time bin after SWD onset, because maximal changes usually occurred at this time.

\section{Electrophysiology and laser Doppler}

flowmetry CBF analysis

Data were prepared for analysis by defining (1) baseline as the last $2 \mathrm{~s}$ of artifact-free recording immediately before the SWD onset and (2) SWD as defined above. SWD onset and offset were marked on the EEG signals using Spike 2, as described above, and these times were used for analyzing the simultaneously acquired MUA, LFP, and CBF signals. For MUA, peristimulus time histograms (PSTHs) were constructed in Spike 2 using a template-matching algorithm as described previously (Nersesyan et al., 2004b).

MUA, LFP, and CBF signals were processed further using an in-house program running on a MATLAB platform. For analyzing LFP and MUA signal amplitude during SWD, the root mean square voltage (Vrms) was measured in consecutive non-overlapping $0.5 \mathrm{~s}$ time bins from $2 \mathrm{~s}$ before to $9 \mathrm{~s}$ after SWD onset. Mean CBF signals were also calculated for the same $0.5 \mathrm{~s}$ bins. To show a time course of mean percentage changes for MUA, LFP, or CBF, we plotted [(signal during SWD - mean baseline)/mean baseline] $\times 100$, for consecutive nonoverlapping $0.5 \mathrm{~s}$ intervals from $2 \mathrm{~s}$ before to $9 \mathrm{~s}$ after SWD onset. As with the BOLD and CBV time courses, data across SWD and experimental runs were first pooled by averaging within each animal, and all graphs were then plotted as mean across animals.

To perform statistical analysis across fMRI, electrophysiology, and laser Doppler flow- 
metry time courses, we calculated the mean percentage signal change separately for all imaging ROIs, LFP signal amplitude, MUA signal amplitude, and CBF signals during the $2-4 \mathrm{~s}$ after SWD onset (time of usual maximal signal change). ANOVA with post hoc Bonferroni's correction for multiple comparisons was used to detect differences in AMRI BOLD and $\mathrm{CBV}$ signals from $\mathrm{CPu}, \mathrm{S} 1 \mathrm{BF}$, and thalamus and electrophysiology and $\mathrm{CBF}$ signals from $\mathrm{CPu}, \mathrm{S} 1 \mathrm{BF}$, and VPM. All statistical tests were processed using SPSS 17 (SPSS), and level significance was assessed at $p<0.05$.

For all BOLD, CBV, LFP, MUA, and CBF calculations, results were similar regardless of whether statistics were performed across SWD episodes, experimental runs, or animals. We chose the more conservative approach of using number of animals as our sample size, pooling first by averaging across SWD and across experimental runs within each animal, and then performing calculations across animals.

\section{Results \\ Mapping BOLD and CBV signal changes during spike-wave seizures}

We first used BOLD fMRI to map cortical and subcortical regions showing increases and decreases during SWD. We analyzed BOLD fMRI data from a total of 1856 SWD events from 45 experimental runs in $22 \mathrm{WAG} / \mathrm{Rij}$ rats. BOLD fMRI during SWD did not involve the whole brain uniformly but rather showed both increases and decreases in specific cortical and subcortical brain regions (Fig. 1). Based on $t$ maps from all animals, we found intense bilateral increases in S1BF (100\%, or 22 of 22 rats), thalamus $(100 \%$, or 22 of 22 rats), anterior cingulate $(91 \%$, or 20 of 22 rats), posterior cingulate/retrosplenial cortex ( $86 \%$, or 19 of 22 rats), and superior colliculi ( $41 \%$, or 9 of 22 rats) (Table 1$)$. In addition, there were several other brain regions in which no changes were observed during SWD, including the hippocampus and the primary visual cortex (V1M) (data not shown in Table). We also found prominent and symmetrical bilateral decreases in the BOLD fMRI signal in the $\mathrm{CPu}(81 \%$, or 18 of 22 rats) (Fig. 1, Table 1). Similarly, reanalysis of our previous data with whisker stimulation performed under different anesthesia conditions (Sanganahalli et al., 2008, 2009a) showed fMRI decreases in the $\mathrm{CPu}$ resembling the fMRI decreases during SWD (data not shown).

For comparison, we analyzed pairs of baseline BOLD fMRI images (image pairs during times with no SWD) at similar time intervals and using the same number of images as in the SWD analysis and did not observe significant changes in any brain region (data not shown). To test the bilateral nature of fMRI changes, we compared the mean fMRI signal changes in left and right structures during the first $4 \mathrm{~s}$ after SWD onset and did not find significant differences between the two sides $(p>0.2)$.

Although BOLD fMRI increases during seizures are typically positively correlated with increased blood flow and neuronal activity, BOLD fMRI decreases are less straightforward. For example, we have seen BOLD fMRI decreases accompanied by either increases (Schridde et al., 2008) or decreases (Englot et al., 2008) in CBV, blood flow, and neuronal activity. To begin to investigate this, CBV data were analyzed from a total of 418 SWD events from six experimental runs in five rats. CBV $t$ maps demonstrated regional changes (Fig. 2) that closely resembled BOLD fMRI $t$ maps for both regional increases and decreases. We found bilateral CBV increases in the S1BF (100\%, or 5 of 5 rats), thalamus ( $100 \%$, or 5 of 5 rats), anterior cingulate $(80 \%$, or 4 of 5 rats), and posterior cingulate/retrosplenial cortex $(60 \%$, or 3 of 5 rats $)$ and bilateral decreases in the anterior cingulate $(20 \%$, or 1 of 5 rats), posterior cingulate/retrosplenial cortex (20\%, or 1 of 5 rats), and $\mathrm{CPu}(100 \%$, or 5 of 5 rats) (Table 1$)$.
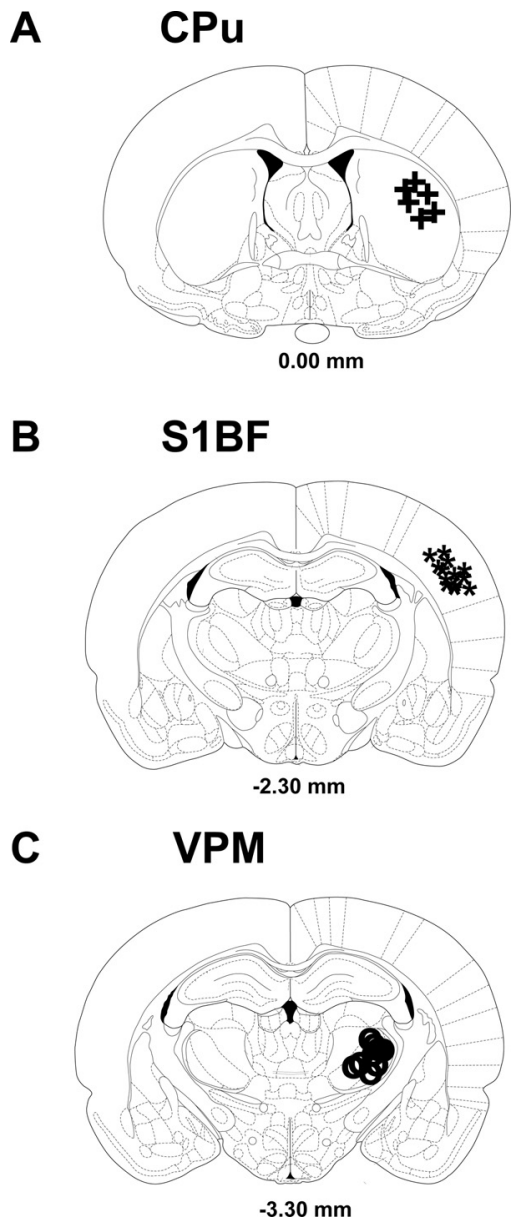

Figure 4. Summary of verification of recording sites on histology. Schematic mapping of recorded sites on coronal brain sections (modified with permission from Paxinos and Watson, 1998) to show location of the tip of combined recording electrode/laser Doppler probe assembly as seen on histological sections from $(\mathrm{Pu}(\boldsymbol{A}), \operatorname{S1BF}(\boldsymbol{B})$, and VPM $(\boldsymbol{C})$.

\section{Time course of BOLD and CBV signals during spike-wave seizures}

To further characterize the pattern of increases and decreases and to compare neuroimaging changes with direct measurements of neurophysiology, we next analyzed the BOLD and CBV time courses for the regions most intensely involved in SWD. This analysis focused on the time courses of signal percentage changes in S1BF, thalamus, and $\mathrm{CPu}$ (Fig. 3A). We also show time courses from hippocampus for comparison. We found that BOLD fMRI signals increased in S1BF and thalamus during SWD events, with signals peaking at 2-4 s after SWD onset and returning toward baseline by $6-8 \mathrm{~s}$ after onset (Fig. $3 B$ ). Conversely, BOLD fMRI signals in $\mathrm{CPu}$ showed decreases with lowest values also occurring at 2-4s after SWD onset and returning toward baseline by $6-8 \mathrm{~s}$ after onset (Fig. 3B). BOLD signal (from 2 to $4 \mathrm{~s}$ after SWD onset) in $\mathrm{CPu}$ was significantly different compared with $\mathrm{S} 1 \mathrm{BF}$ and thalamus $\left(p=5.2 \times 10^{-9}\right.$ and $3.7 \times 10^{-6}$, respectively, with Bonferroni's correction for multiple comparisons), whereas there was no significant difference between signal from S1BF and thalamus (see Fig. $8 A$ ). In the hippocampus, unlike S1BF, VPM, and $\mathrm{CPu}$, we found no significant difference in BOLD signal 2-4 s after SWD onset versus baseline (Fig. $3 B)(p=0.28)$. Although an initial dip (Frostig et al., 1990; Ances, 2004; Suh et al., 2005; Chen-Bee et al., 2007) was not seen in any of the regions even with higher-resolution binning $(0.5 \mathrm{~s})$ of our BOLD time course data 


\section{A Scalp EEG

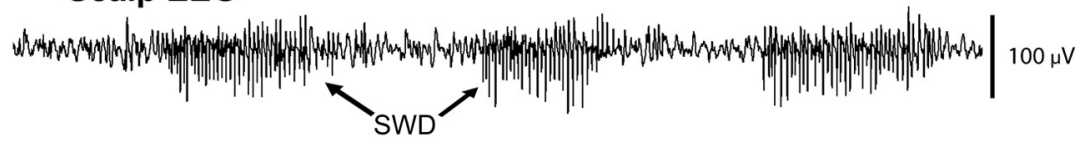
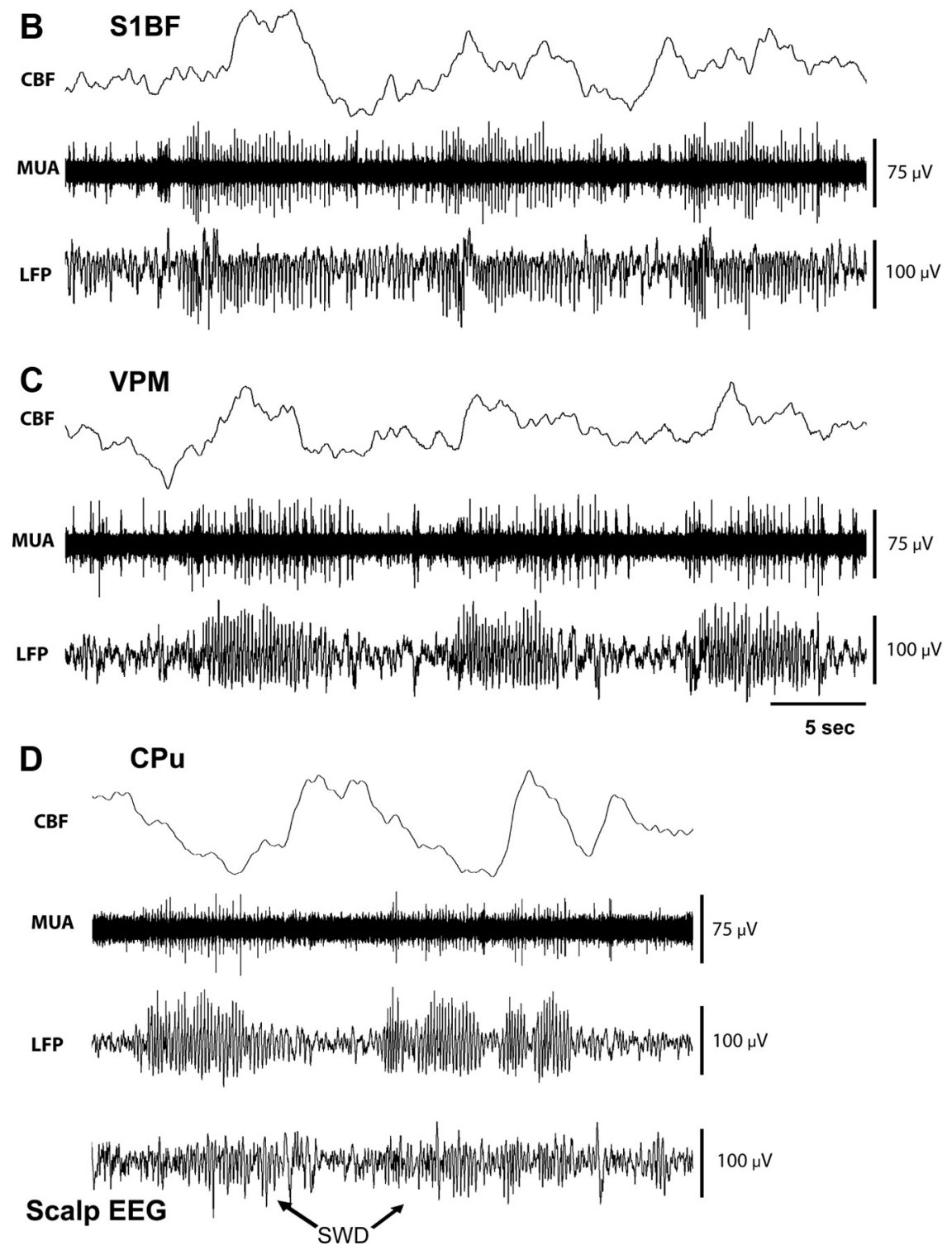

Figure 5. SWDs produce increased electrical activity with increased CBF in cortex and thalamus but decreased CBF in striatum. $A-C$, Example of simultaneous recordings of MUA, LFPs, and laser Doppler CBF from S1BF and VPM, as well as scalp EEG during three consecutive SWD episodes. $A$, Scalp EEG demonstrating three SWD episodes. $\boldsymbol{B}$, Recordings from S1BF during SWDs show increases in multiunit activity and local field potentials, followed by an increase in CBF. C, Recordings from VPM during SWD show increases in local field potentials and multiunit activity, followed by an increase in CBF. D, Example from a different animal of simultaneous recordings of scalp EEG, MUA, LFP, and CBF from CPu during SWD episodes (simultaneous S1BF recordings for this animal are not shown). Recordings from (Pu during SWD show increases in LFP, subtle increases in MUA, and large decreases in CBF. Recordings of $(B F$ (in $\boldsymbol{B}-\boldsymbol{D}$ ) are displayed with 0.5 s time bins.

(data not shown), a transient initial dip could have been missed with these methods, unlike higher temporal resolution techniques such as optical imaging.

Time courses of percentage change in CBV similarly showed increases in S1BF and thalamus and decreases in $\mathrm{CPu}$, with maximal deflections occurring 2-4s after SWD onset (Fig. 3C). Mean CBV change (from 2 to $4 \mathrm{~s}$ after SWD onset) in CPu was significantly different compared with S1BF and thalamus $(p=8.02 \times$
$10^{-5}$ and $9.57 \times 10^{-5}$, respectively, with Bonferroni's correction for multiple comparisons), whereas no significant difference was observed between S1BF and thalamus (see Fig. $8 B$ ). For comparison, the hippocampus again showed no significant signal change $2-4 \mathrm{~s}$ after SWD onset versus baseline (Fig. $3 C)(p=0.84)$.

Neuronal activity and CBF during SWD show paradoxical changes in $\mathrm{CPu}$ We next used direct electrophysiological and $\mathrm{CBF}$ measurements to investigate the basis of cortical and thalamic fMRI increases, as well as $\mathrm{CPu}$ fMRI decreases. In particular, if normal neurovascular coupling operates under these conditions, we would expect to see neuronal activity increases in cortex and thalamus; conversely, neuronal activity decreases would be expected in $\mathrm{CPu}$. Electrophysiological and $\mathrm{CBF}$ recordings were performed during 704 SWDs from S1BF $(n=13$ animals in 23 experimental runs), 436 SWDs from VPM $(n=9$ animals in 14 experimental runs), and 268 SWDs from $\mathrm{CPu}(n=6$ animals in 8 experimental runs). For simultaneous recordings of electrophysiology and CBF changes with laser Doppler flowmetry, we focused our studies in the $\mathrm{S} 1 \mathrm{BF}, \mathrm{VPM}$, and $\mathrm{CPu}$ because these regions were found to be maximally involved during SWDs in our fMRI studies. Electrode locations verified histologically (Fig. 4) matched reasonably well with ROIs used for image analysis (Fig. 3A).

During SWDs (Fig. 5A, scalp EEG), we observed that S1BF and VPM both showed immediate increases in MUA and LFP, followed by increases in CBF peaking a few seconds after SWD onset (Fig. $5 B, C)$. In contrast, in $\mathrm{CPu}$, we observed a large increase in LFP and a smaller increase in MUA, accompanied by prominent CBF decreases during SWDs (Fig. $5 D)$. Because $\mathrm{CBF}$ and neuronal activity changes are in opposite directions in $\mathrm{CPu}$, these findings are not compatible with normal neurovascular coupling. PSTHs of unit activity around the seizures from $\mathrm{CPu}, \mathrm{S} 1 \mathrm{BF}$, and VPM (Fig. 6A-C) show large increases in neuronal firing in S1BF and VPM, whereas $\mathrm{CPu}$ showed a relatively small increase.

In group time courses, again all signals increased in S1BF and VPM, whereas signals diverged in CPu. Thus, S1BF and VPM showed increases in percentage change LFP and MUA signal amplitude during SWDs (Fig. $7 A, C$ ), followed by mean CBF increases in S1BF and VPM (Fig. 7E). Surprisingly, $\mathrm{CPu}$ showed a small increase in MUA and a disproportionately large increase in LFP signal amplitude (Fig. $7 \mathrm{~A}, \mathrm{C}$ ). These electrophysiological changes in $\mathrm{CPu}$ were associated with a large decrease in CBF (Fig. 7E). One possible concern is that calculating 
A S1BF

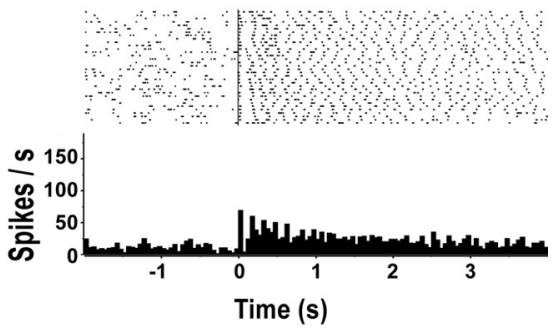

B VPM

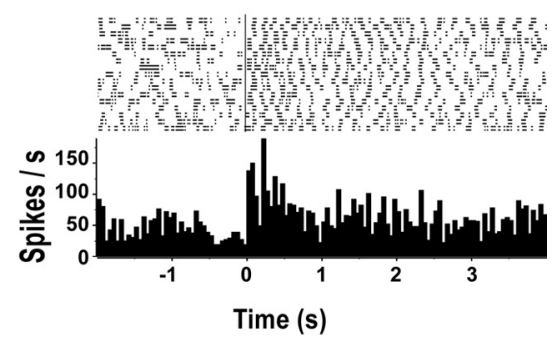

C $\mathrm{CPu}$

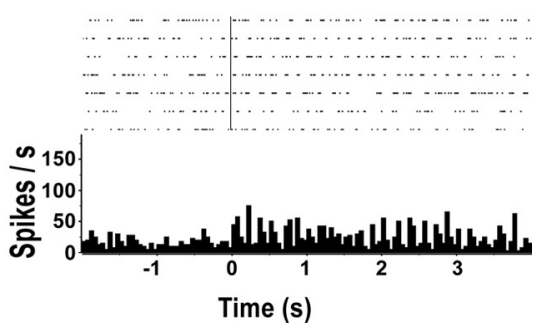

Figure 6. PSTHs of unit activity. Examples of PSTHs around spike-wave seizures recorded from CPu ( $n=7$ SWDs), S1BF ( $n=34$ SWDs), and VPM ( $n=17$ SWDs). Vertical lines in the raster plots (top) indicate seizure onset. PSTH bins (bottom) are $50 \mathrm{~ms}$.

percentage change in LFP and MUA signal amplitude from baseline may distort the relative amplitudes because the baseline signal is not the same in all regions. Therefore, we also plotted mean LFP and MUA signal amplitude without dividing by baseline (Fig. $7 B, D$ ) and still found increases in electrophysiology signals in all regions with similar relative changes to those seen with the percentage calculations (Fig. $7 A, C$ ).

The CBF signal (from 2 to $4 \mathrm{~s}$ after SWD onset) in $\mathrm{CPu}$ was significantly decreased compared with the CBF signal in S1BF and VPM $\left(p=1.4 \times 10^{-7}\right.$ and $6.1 \times 10^{-8}$, respectively, with Bonferroni's correction for multiple comparisons) (Fig. 8C). CBF signals were not significantly different between S1BF and VPM.

Increases in LFP signal amplitude in $\mathrm{CPu}$ were significantly higher compared with S1BF and VPM $\left(p=1.1 \times 10^{-8}\right.$ and $4.4 \times$ $10^{-8}$, respectively, Bonferroni's corrected) (Fig. $8 D$ ). LFP signal amplitude (from 2 to $4 \mathrm{~s}$ after SWD onset) was not significantly different in VPM compared with S1BF $(p=0.32)$.

MUA signal amplitude (from 2 to $4 \mathrm{~s}$ after SWD onset) in $\mathrm{CPu}$ was significantly lower compared with S1BF and VPM $(p=7.9 \times$ $10^{-4}$ and $5.5 \times 10^{-5}$, respectively, Bonferroni's corrected), whereas no significant difference was observed in MUA signal amplitude between S1BF and VPM (Fig. 8E).

Normal somatosensory stimulation is also associated with paradoxical changes in $\mathrm{CPu}$

We next asked whether the observed changes were specific to SWDs or may also occur with normal somatosensory stimula-
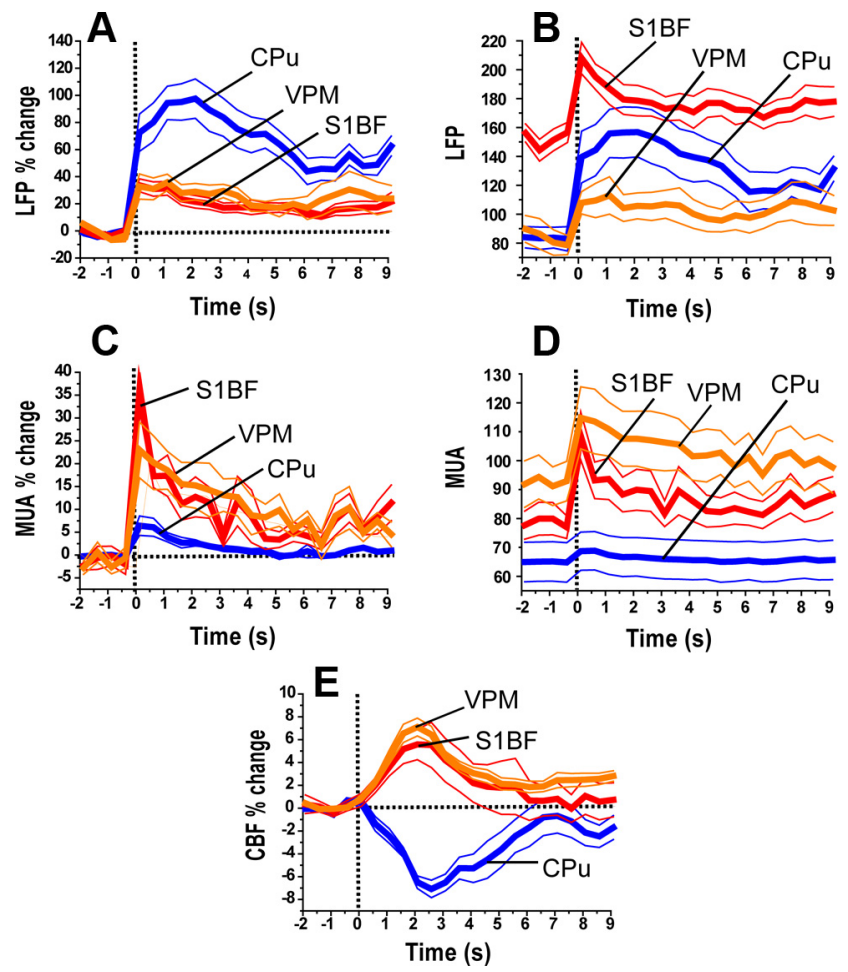

Figure 7. Mean time courses of electrophysiology and CBF measurements during SWDs. Signals are shown in S1BF, VPM, and CPu. Time courses represent percentage change in LFP signal amplitude (Vrms) compared with $2 \mathrm{~s}$ baseline (A), LFP signal amplitude (Vrms) without percentage change calculation $(\boldsymbol{B})$, percentage change in MUA signal amplitude (Vrms) compared with $2 \mathrm{~s}$ baseline $(\boldsymbol{C})$, MUA signal amplitude (Vrms) without percentage change calculation (D), and percentage change in (BF signal (E). Recording sites (Fig. 4) were matched to ROls chosen in analyzing $f M R I$ signal time courses (Fig. 3). During seizures, LFPs $(\boldsymbol{A}, \boldsymbol{B})$ and MUA $(\boldsymbol{C}, \boldsymbol{D})$ showed a marked increase in signal amplitude in S1BF and VPM, accompanied by strong increases in CBF (E). In contrast, CPu recordings showed intense signal increases for $\operatorname{LFP}(\boldsymbol{A}, \boldsymbol{B})$ associated with a small increase in $\operatorname{MUA}(\boldsymbol{C}, \boldsymbol{D})$ and a large decrease in $\mathrm{CBF}(\boldsymbol{E})$ during SWDs. Note that $y$-axes in $\boldsymbol{A}-\boldsymbol{E}$ are not drawn to the same scale. Time courses are displayed as mean $\pm \mathrm{SEM}$, with 0.5 s time bins. Data are from S1BF ( $n=13$ animals), VPM ( $n=9$ animals), and CPu ( $n=$ 6 animals). Vertical line at time $=0$ marks SWD onset.

tion. We obtained electrophysiology and CBF data from a total of 104 whisker stimulations, 41 recorded from S1BF $(n=13$ animals in 23 experimental runs), 31 from VPM ( $n=8$ animals in 13 experimental runs), and 32 from $\mathrm{CPu}(n=6$ animals in 8 experimental runs). In agreement with a previous report (Nersesyan et al., 2004b), we found similar but slightly greater increases in mean CBF and MUA signals in S1BF with whisker stimulation (Fig. $8 F, G$ ) compared with SWDs (Fig. 8C,E). LFP could not be analyzed reliably because of low-frequency voltage artifacts introduced by the whisker stimulus. In VPM, we also observed increases in MUA and CBF during whisker stimulations that were generally similar to SWDs (Fig. $8 C-G$ ). Interestingly, $\mathrm{CPu}$ also showed similar changes during whisker stimulation and SWDs. Thus, we observed CBF decreases in $\mathrm{CPu}$ during whisker stimulation (Fig. $8 F$ ) despite the presence of MUA increases (Fig. $8 G$ ). Therefore, the paradoxical CBF decreases observed during MUA increases in $\mathrm{CPu}$ are not specific to SWDs, because they are also present during normal whisker stimulation in WAG/Rij rats, in agreement with previous somatosensory stimuli in non-epileptic rats (Shih et al., 2009, 2011).

In summary, neuroimaging measures such as BOLD, CBV, and CBF during SWD and whisker stimulation showed increases in cortex and thalamus but decreases in $\mathrm{CPu}$ (Fig. $8 A-C, F$ ). In contrast, electrophysiological measurements including LFP 

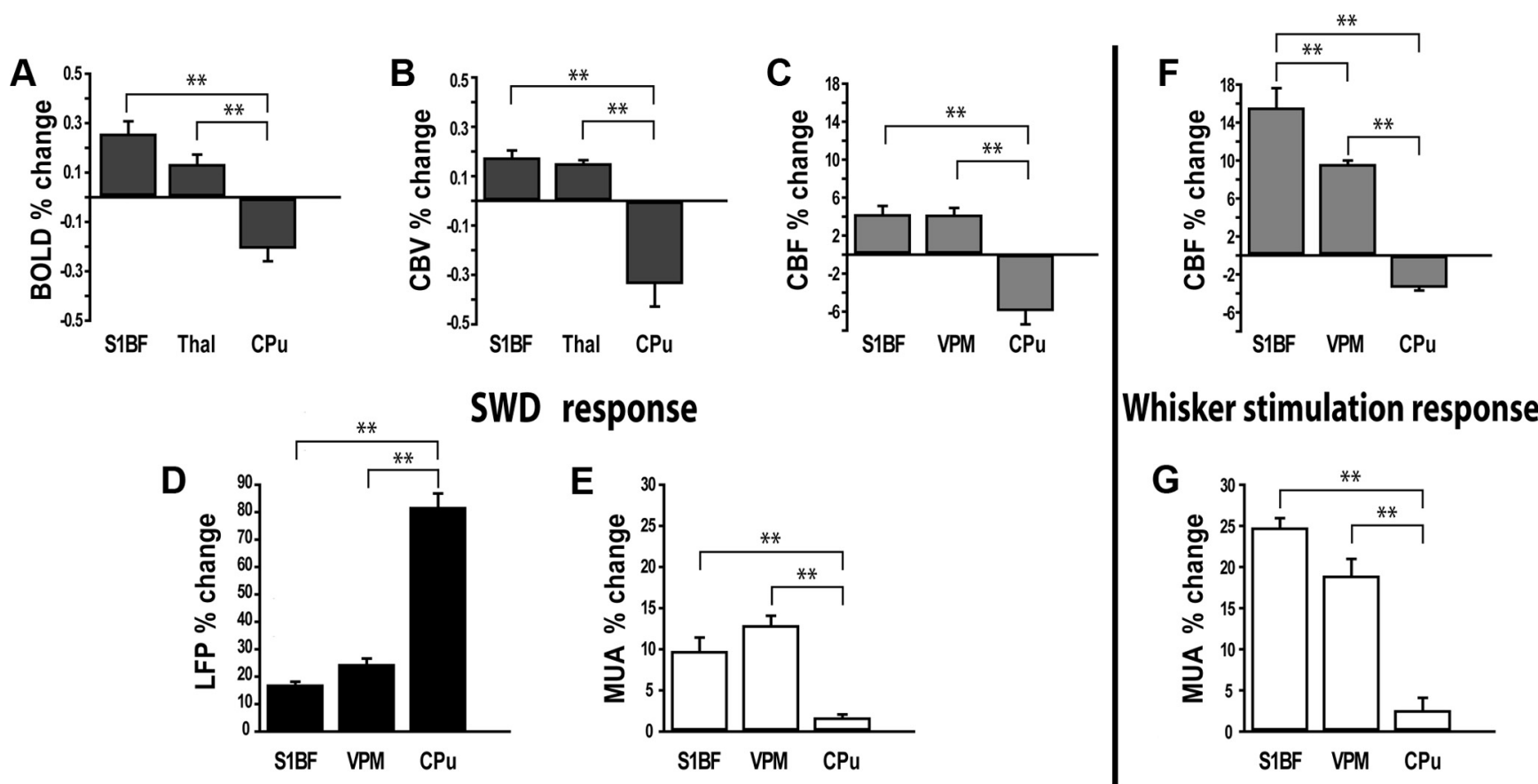

Figure 8. Summary of neuroimaging-related (top row) and electrophysiology (bottom row) signal changes during SWDs and whisker stimulation. Values are mean percentage change, comparing the baseline signal with 2-4s after onset of SWDs or whisker stimulation. $\boldsymbol{A}$, BOLD during SWD; $\boldsymbol{B}$, CBV during SWD; C, CBF during SWD; $\boldsymbol{D}$, LFP signal amplitude (Vrms) during SWD; $\boldsymbol{E}$, MUA signal amplitude (Vrms) during SWD. $\boldsymbol{A}$-Cand $\boldsymbol{D}, \boldsymbol{E}$ are from same data shown in Figures 3 and 7, respectively. $\boldsymbol{F}$, CBF during whisker stimulation. $\boldsymbol{G}$, MUA signal amplitude (Vrms) during whisker stimulation. Overall across conditions, S1BF and VPM showed increases in all measurements (BOLD, CBV, CBF, LFP, and MUA). CPu showed large increases in LFP, a small increase in MUA, but a large decrease in BOLD, CBV, and CBF. ${ }^{* *} p<0.01$, ANOVA with post hoc Bonferroni's correction for multiple comparisons. Note that $y$-axes are drawn to the same scale for similar measurements for $\boldsymbol{A}$ and $\boldsymbol{B}(\mathrm{BOLD}, \mathrm{CBV})$, for $\boldsymbol{C}$ and $\boldsymbol{F}(\mathrm{CBF})$, and for $\boldsymbol{E}$ and $\boldsymbol{G}(\mathrm{MUA})$. Thal, Thalamus.

and MUA showed increased activity in all three regions (Fig. $8 D, E, G)$.

\section{Discussion}

We investigated the relationship between neuroimaging signals and electrophysiology in cortical and subcortical structures in the WAG/Rij rat. In the cortex, fMRI neuroimaging-related signals such as BOLD, CBV, and CBF agreed with electrophysiological measurements of MUA and LFP. Similar agreement between neuroimaging and electrophysiology was obtained in one important subcortical region, the thalamic VPM. However, in another subcortical structure, the caudate-putamen, fMRI BOLD, CBV, and laser Doppler CBF measurements all showed strong decreases, whereas electrophysiological measurements showed increased neuronal activity. Despite neuroimaging signal decreases in $\mathrm{CPu}$, we saw large increases in LFP along with smaller increases in MUA in the same region. Similar relationships were found both during SWDs and normal whisker stimulation. These findings suggest that neurovascular coupling operates in the expected manner in the cortical S1BF and subcortical VPM of the thalamus, but we observed a striking reverse coupling of neuronal and vascular activity in $\mathrm{CPu}$.

Previous work suggests that fMRI increases in the cortex during SWD are closely related to changes in neuronal activity (Nersesyan et al., 2004a,b). In agreement with the present findings, these studies found increases in BOLD, CBF, and electrophysiological measurements in S1BF, whereas other regions, such as the occipital cortex, were relatively spared. Focal cortical involvement may be important for the pathophysiology (Meeren et al., 2002, 2005; Klein et al., 2004) and chronic brain changes in spikewave epilepsy (Blumenfeld et al., 2008; Caplan et al., 2009; Chahboune et al., 2009). Numerous other studies also support the general agreement of fMRI and neuronal activity increases in the cortex during normal sensory activation (Logothetis et al., 2001; Smith et al., 2002; Maandag et al., 2007; Sanganahalli et al., 2009b). Human fMRI studies during SWD have shown a combination of regional cortical increases and decreases (Archer et al., 2003; Salek-Haddadi et al., 2003; Aghakhani et al., 2004; Gotman et al., 2005; Labate et al., 2005; Hamandi et al., 2006; Laufs et al., 2006; Hawco et al., 2007; Moeller et al., 2008a,b; Vaudano et al., 2009; Bai et al., 2010; Berman et al., 2010). Recent work suggests that this may be related to the variable time course of fMRI signals in different brain regions during human SWDs (Moeller et al., 2008b; Bai et al., 2010). It will be important to investigate further the neuronal basis of variable cortical fMRI changes during SWDs, although because rat models show mainly cortical increases (Tenney et al., 2003, 2004; Nersesyan et al., 2004a; David et al., 2008), additional novel animal models may be helpful.

Human fMRI studies have shown consistent fMRI increases in the thalamus during SWDs (Salek-Haddadi et al., 2003; Aghakhani et al., 2004; Gotman et al., 2005; Labate et al., 2005; Hamandi et al., 2006; Laufs et al., 2006; Hawco et al., 2007; Moeller et al., 2008a,b; Vaudano et al., 2009; Bai et al., 2010; Berman et al., 2010). Previous work has not directly related thalamic neuroimaging signals to electrophysiology during SWD. In the present study, we found that fMRI, CBV, and CBF increases in the VPM of the thalamus are concordant with increases in MUA and LFP signals in the same region. Because the thalamus is a complex structure, including regions of mainly excitatory or mainly inhibitory neurons (Steriade et al., 1997), additional detailed studies will be crucial to relate neuroimaging and electrophysiology signal in other thalamic regions during SWD. 
The caudate-putamen has been reported to play a modulatory role in the control of SWDs (Deransart et al., 1998). Interestingly, the caudate-putamen shows mainly fMRI decreases in human studies of SWDs (Moeller et al., 2008a,b; Bai et al., 2010; Berman et al., 2010). In a human study, the basal ganglia have also shown fMRI deactivations during acupuncture (Hui et al., 2000). Decreases in fMRI signals could arise from several mechanisms, including primary neuronal, primary vascular, or neurovascular coupling alterations (Blumenfeld, 2007; Motelow and Blumenfeld, 2009). Under normal conditions, primary neuronal mechanisms are thought to drive most BOLD fMRI signal decreases, when a decrease in neuronal activity and oxygen consumption occurs in the setting of an even greater reduction in CBF (Shmuel et al., 2002, 2006; Stefanovic et al., 2004; Devor et al., 2007). A primary neuronal mechanism is likely to explain BOLD fMRI decreases seen during cortical slow oscillations in limbic seizures, in which cortical neuronal firing, $\mathrm{CBF}$, and $\mathrm{CBV}$ all decrease (Englot et al., 2008, 2009). However, in our present measurements from $\mathrm{CPu}$, we observed $\mathrm{BOLD}, \mathrm{CBV}$, and $\mathrm{CBF}$ decreases along with neuronal electrical activity increases, so that a primary decrease in neuronal activity is not the mechanism. In a tonicclonic seizure model, altered neurovascular coupling produced BOLD decreases in the hippocampus, because large increases in neuronal activity and oxygen consumption exceeded increases in CBF and CBV (Schridde et al., 2008). Altered neurovascular coupling has also been reported in other forms of epilepsy (Fink et al., 1996; Suh et al., 2006). However, the present results do not reflect an insufficient increase in CBF in the face of neuronal activity increases. Rather, the $\mathrm{CPu}$ shows an opposite change, suggesting that a primary vascular decrease in $\mathrm{CBF}$ is the most likely mechanism for the reduced BOLD signals in $\mathrm{CPu}$.

A primary vascular decrease in $\mathrm{CBF}$ could arise either from the "vascular steal" phenomenon or vascular spasm (Harel et al., 2002). Factors in favor of vascular steal include the large BOLD and CBV increases we observed in cortical sensory regions neighboring $\mathrm{CPu}$ and the fact that a proportion of the arterial blood supply to the sensory cortex and CPu is shared (Scremin, 2004). It has been speculated previously that decreased CBF in an area adjacent to a seizure focus may be attributable to vascular steal phenomena (Zhao et al., 2009).

However, other studies of the $\mathrm{CPu}$ suggest that vasoconstriction (rather than vascular steal) could play a dominant role in producing BOLD decreases despite increases in neuronal activity. Cerebral blood volume in $\mathrm{CPu}$ is modulated by dopamine, with increased CBV mediated through $\mathrm{D}_{1} / \mathrm{D}_{5}$ receptors and decreased CBV mediated through $\mathrm{D}_{2} / \mathrm{D}_{3}$ receptors (Choi et al., 2006). Recent studies during somatosensory stimulation have shown decreased fMRI BOLD, CBV, and CBF despite increased neuronal activity in the $\mathrm{CPu}$, similar to our results; moreover, it was found that the vascular decreases could be blocked by a $\mathrm{D}_{2}$ receptor antagonist (Shih et al., 2009, 2011). These findings suggest that $\mathrm{D}_{2}$ receptor activation by dopamine release during SWDs and normal somatosensory stimulation may produce direct vasoconstriction, leading to decreases in BOLD, CBF, and CBV despite local increases in neuronal activity.

Another important point to consider is the significance of large LFP signals during synchronized rhythmic neuronal activity, which may not reflect neuronal energy metabolism as directly as under conditions of transient sensory stimulation. For example, in a previous study, we found large-amplitude LFP increases during cortical slow oscillations in rat limbic seizures, which were accompanied by decreases in mean rate of neuronal firing, as well as decreases in BOLD, CBF, CBV, and cerebral metabolic rate of oxygen consumption (Englot et al., 2008). In the present case, we observed large-amplitude LFP increases in CPu during SWDs, along with only small increases in mean rate of neuronal firing. As already noted, the accompanying decreases in BOLD, CBV, and CBF are likely attributable to primary vascular mechanisms. However, the discrepancy between LFP and MUA signal changes may be attributable to strong activation of striatal inhibitory neurons during SWDs, giving rise to a large increase in synaptic activity but only a small increase in mean rate of neuronal firing, as reported previously in a similar model (Slaght et al., 2004).

In summary, during SWDs in the WAG/Rij rat, we found transient focal increases in BOLD and CBV signals matched by electrophysiology and CBF recordings in regions known to be intensely involved in SWDs, such as the somatosensory barrel cortex and thalamus. Surprisingly, we found that consistent $\mathrm{BOLD}, \mathrm{CBV}$, and CBF decreases in caudate-putamen were associated with a large increase in local field potentials and a small increase in multiunit activity. Similar results were found with both SWD and normal somatosensory stimulation using CBF and electrophysiology measurements in these regions. These findings suggest that, in SWDs, as in normal brain activity, corticothalamic oxygen delivery increases in regions of increased neuronal activity. However, in the caudate-putamen, vascular signals decrease despite an increase in neuronal activity. These results support conventional interpretation of cortical and thalamic fMRI signals but raise a cautionary note about fMRI signals from caudate-putamen and possibly other subcortical regions as well, warranting additional investigation. Thus, fMRI and electrophysiology measurements generally agree in the somatosensory cortex and thalamus. However, in the striatum, these signals have had a mutual parting of the ways.

\section{References}

Aghakhani Y, Bagshaw AP, Bénar CG, Hawco C, Andermann F, Dubeau F, Gotman J (2004) fMRI activation during spike and wave discharges in idiopathic generalized epilepsy. Brain 127:1127-1144.

Ances BM (2004) Coupling of changes in cerebral blood flow with neural activity: what must initially dip must come back up. J Cereb Blood Flow Metab 24:1-6.

Archer JS, Abbott DF, Waites AB, Jackson GD (2003) fMRI “deactivation" of the posterior cingulate during generalized spike and wave. Neuroimage 20:1915-1922.

Bai X, Vestal M, Berman R, Negishi M, Spann M, Vega C, Desalvo M, Novotny EJ, Constable RT, Blumenfeld H (2010) Dynamic time course of typical childhood absence seizures: EEG, behavior, and functional magnetic resonance imaging. J Neurosci 30:5884-5893.

Benner T, van der Kouwe AJ, Kirsch JE, Sorensen AG (2006) Real-time RF pulse adjustment for B0 drift correction. Magn Reson Med 56:204-209.

Berman R, Negishi M, Vestal M, Spann M, Chung MH, Bai X, Purcaro M, Motelow JE, Danielson N, Dix-Cooper L, Enev M, Novotny EJ, Constable RT, Blumenfeld H (2010) Simultaneous EEG, fMRI, and behavior in typical childhood absence seizures. Epilepsia 51:2011-2022.

Blumenfeld H (2007) Functional MRI studies of animal models in epilepsy. Epilepsia 48 [Suppl 4]:18-26.

Blumenfeld H, Klein JP, Schridde U, Vestal M, Rice T, Khera DS, Bashyal C, Giblin K, Paul-Laughinghouse C, Wang F, Phadke A, Mission J, Agarwal RK, Englot DJ, Motelow J, Nersesyan H, Waxman SG, Levin AR (2008) Early treatment suppresses the development of spike-wave epilepsy in a rat model. Epilepsia 49:400-409.

Caplan R, Levitt J, Siddarth P, Wu KN, Gurbani S, Sankar R, Shields WD (2009) Frontal and temporal volumes in childhood absence epilepsy. Epilepsia 50:2466-2472.

Chahboune H, Mishra AM, DeSalvo MN, Staib LH, Purcaro M, Scheinost D, Papademetris X, Fyson SJ, Lorincz ML, Crunelli V, Hyder F, Blumenfeld H (2009) DTI abnormalities in anterior corpus callosum of rats with spike-wave epilepsy. Neuroimage 47:459-466.

Chen-Bee CH, Agoncillo T, Xiong Y, Frostig RD (2007) The triphasic intrinsic signal: implications for functional imaging. J Neurosci 27:4572-4586. 
Choi JK, Chen YI, Hamel E, Jenkins BG (2006) Brain hemodynamic changes mediated by dopamine receptors: role of the cerebral microvasculature in dopamine-mediated neurovascular coupling. Neuroimage 30:700-712.

Coenen AM, Van Luijtelaar EL (1987) The WAG/Rij rat model for absence epilepsy: age and sex factors. Epilepsy Res 1:297-301.

Coenen AM, Van Luijtelaar EL (2003) Genetic animal models for absence epilepsy: a review of the WAG/Rij strain of rats. Behav Genet 33:635-655.

Coenen AM, Drinkenburg WH, Inoue M, van Luijtelaar EL (1992) Genetic models of absence epilepsy, with emphasis on the WAG/Rij strain of rats. Epilepsy Res 12:75-86.

David O, Guillemain I, Saillet S, Reyt S, Deransart C, Segebarth C, Depaulis A (2008) Identifying neural drivers with functional MRI: an electrophysiological validation. PLoS Biol 6:2683-2697.

Deransart C, Vercueil L, Marescaux C, Depaulis A (1998) The role of basal ganglia in the control of generalized absence seizures. Epilepsy Res 32:213-223.

DeSalvo MN, Schridde U, Mishra AM, Motelow JE, Purcaro MJ, Danielson N, Bai X, Hyder F, Blumenfeld H (2010) Focal BOLD fMRI changes in bicuculline-induced tonic-clonic seizures in the rat. Neuroimage 50:902-909.

D'Esposito M, Deouell LY, Gazzaley A (2003) Alterations in the BOLD fMRI signal with ageing and disease: a challenge for neuroimaging. Nat Rev Neurosci 4:863-872.

Devor A, Tian P, Nishimura N, Teng IC, Hillman EM, Narayanan SN, Ulbert I, Boas DA, Kleinfeld D, Dale AM (2007) Suppressed neuronal activity and concurrent arteriolar vasoconstriction may explain negative blood oxygenation level-dependent signal. J Neurosci 27:4452-4459.

Englot DJ, Mishra AM, Mansuripur PK, Herman P, Hyder F, Blumenfeld H (2008) Remote effects of focal hippocampal seizures on the rat neocortex. J Neurosci 28:9066-9081.

Englot DJ, Modi B, Mishra AM, DeSalvo M, Hyder F, Blumenfeld H (2009) Cortical deactivation induced by subcortical network dysfunction in limbic seizures. J Neurosci 29:13006-13018.

Fink GR, Pawlik G, Stefan H, Pietrzyk U, Wienhard K, Heiss WD (1996) Temporal lobe epilepsy: evidence for interictal uncoupling of blood flow and glucose metabolism in temporomesial structures. J Neurol Sci 137:28-34.

Foerster BU, Tomasi D, Caparelli EC (2005) Magnetic field shift due to mechanical vibration in functional magnetic resonance imaging. Magn Reson Med 54:1261-1267.

Frostig RD, Lieke EE, Ts'o DY, Grinvald A (1990) Cortical functional architecture and local coupling between neuronal activity and the microcirculation revealed by in vivo high-resolution optical imaging of intrinsic signals. Proc Natl Acad Sci U S A 87:6082-6086.

Gotman J, Grova C, Bagshaw A, Kobayashi E, Aghakhani Y, Dubeau F (2005) Generalized epileptic discharges show thalamocortical activation and suspension of the default state of the brain. Proc Natl Acad Sci U S A 102:15236-15240.

Hamandi K, Salek-Haddadi A, Laufs H, Liston A, Friston K, Fish DR, Duncan JS, Lemieux L (2006) EEG-fMRI of idiopathic and secondarily generalized epilepsies. Neuroimage 31:1700-1710.

Harel N, Lee SP, Nagaoka T, Kim DS, Kim SG (2002) Origin of negative blood oxygenation level-dependent fMRI signals. J Cereb Blood Flow Metab 22:908-917.

Hawco CS, Bagshaw AP, Lu Y, Dubeau F, Gotman J (2007) BOLD changes occur prior to epileptic spikes seen on scalp EEG. Neuroimage 35:1450-1458.

Hui KK, Liu J, Makris N, Gollub RL, Chen AJ, Moore CI, Kennedy DN, Rosen BR, Kwong KK (2000) Acupuncture modulates the limbic system and subcortical gray structures of the human brain: evidence from fMRI studies in normal subjects. Hum Brain Mapp 9:13-25.

Hyder F (2004) Driving changes in CMRo2 from calibrated fMRI. In: Brain energetics and neuronal activity: applications to fMRI and medicine (Shulman RG, Rothman DL, eds), pp 147-171. New York: Wiley.

Kennan RP, Scanley BE, Innis RB, Gore JC (1998) Physiological basis for BOLD MR signal changes due to neuronal stimulation: separation of blood volume and magnetic susceptibility effects. Magn Reson Med 40:840-846.

Klein JP, Khera DS, Nersesyan H, Kimchi EY, Waxman SG, Blumenfeld H (2004) Dysregulation of sodium channel expression in cortical neurons in a rodent model of absence epilepsy. Brain Res 1000:102-109.

Labate A, Briellmann RS, Abbott DF, Waites AB, Jackson GD (2005) Typical childhood absence seizures are associated with thalamic activation. Epileptic Disord 7:373-377.

Lasoń W, Przewłocka B, Van Luijtelaar EL, Coenen AM, Przewłocki R (1992) Endogenous opioid peptides in brain and pituitary of rats with absence epilepsy. Neuropeptides 21:147-152.

Laufs H, Lengler U, Hamandi K, Kleinschmidt A, Krakow K (2006) Linking generalized spike-and-wave discharges and resting state brain activity by using EEG/fMRI in a patient with absence seizures. Epilepsia 47:444-448.

Logothetis NK, Pauls J, Augath M, Trinath T, Oeltermann A (2001) Neurophysiological investigation of the basis of the fMRI signal. Nature 412:150-157.

Maandag NJ, Coman D, Sanganahalli BG, Herman P, Smith AJ, Blumenfeld H, Shulman RG, Hyder F (2007) Energetics of neuronal signaling and fMRI activity. Proc Natl Acad Sci U S A 104:20546-20551.

Meeren HK, Pijn JP, Van Luijtelaar EL, Coenen AM, Lopes da Silva FH (2002) Cortical focus drives widespread corticothalamic networks during spontaneous absence seizures in rats. J Neurosci 22:1480-1495.

Meeren H, van Luijtelaar G, Lopes da Silva F, Coenen A (2005) Evolving concepts on the pathophysiology of absence seizures: the cortical focus theory. Arch Neurol 62:371-376.

Moeller F, Siebner HR, Wolff S, Muhle H, Granert O, Jansen O, Stephani U, Siniatchkin M (2008a) Simultaneous EEG-fMRI in drug-naive children with newly diagnosed absence epilepsy. Epilepsia 49:1510-1519.

Moeller F, Siebner HR, Wolff S, Muhle H, Boor R, Granert O, Jansen O, Stephani U, Siniatchkin M (2008b) Changes in activity of striatothalamo-cortical network precede generalized spike wave discharges. Neuroimage 39:1839-1849.

Motelow JE, Blumenfeld H (2009) Functional neuroimaging of spike-wave seizures. Methods Mol Biol 489:189-209.

Nersesyan H, Hyder F, Rothman DL, Blumenfeld H (2004a) Dynamic fMRI and EEG recordings during spike-wave seizures and generalized tonicclonic seizures in WAG/Rij rats. J Cereb Blood Flow Metab 24:589-599.

Nersesyan H, Herman P, Erdogan E, Hyder F, Blumenfeld H (2004b) Relative changes in cerebral blood flow and neuronal activity in local microdomains during generalized seizures. J Cereb Blood Flow Metab 24:1057-1068

Ogawa S, Menon RS, Tank DW, Kim SG, Merkle H, Ellermann JM, Ugurbil K (1993) Functional brain mapping by blood oxygenation level-dependent contrast magnetic resonance imaging. Biophys J 64:803-812.

Ogawa S, Menon RS, Kim SG, Ugurbil K (1998) On the characteristics of functional magnetic resonance imaging of the brain. Annu Rev Biophys Biomol Struct 27:447-474.

Paxinos G, Watson C (1998) The rat brain in stereotaxic coordinates, Ed 4. San Diego: Academic.

Pinault D, Leresche N, Charpier S, Deniau JM, Marescaux C, Vergnes M, Crunelli V (1998) Intracellular recordings in thalamic neurones during spontaneous spike and wave discharges in rats with absence epilepsy. J Physiol 509:449-456.

Salek-Haddadi A, Lemieux L, Merschhemke M, Friston KJ, Duncan JS, Fish DR (2003) Functional magnetic resonance imaging of human absence seizures. Ann Neurol 53:663-667.

Sanganahalli BG, Herman P, Hyder F (2008) Frequency-dependent tactile responses in rat brain measured by functional MRI. NMR Biomed 21:410-416

Sanganahalli BG, Bailey CJ, Herman P, Hyder F (2009a) Tactile and nontactile sensory paradigms for fMRI and neurophysiologic studies in rodents. Methods Mol Biol 489:213-242.

Sanganahalli BG, Herman P, Blumenfeld H, Hyder F (2009b) Oxidative neuroenergetics in event-related paradigms. J Neurosci 29:1707-1718.

Schridde U, Khubchandani M, Motelow JE, Sanganahalli BG, Hyder F, Blumenfeld H (2008) Negative BOLD with large increases in neuronal activity. Cereb Cortex 18:1814-1827.

Scremin OU (2004) The rat nervous system, Ed 3 (Paxinos G, ed), pp 1167 1202. San Diego: Academic.

Shih YY, Chen CC, Shyu BC, Lin ZJ, Chiang YC, Jaw FS, Chen YY, Chang C (2009) A new scenario for negative functional magnetic resonance imaging signals: endogenous neurotransmission. J Neurosci 29:3036-3044.

Shih YY, Wey HY, De La Garza BH, Duong TQ (2011) Striatal and cortical BOLD, blood flow, blood volume, oxygen consumption, and glucose consumption changes in noxious forepaw electrical stimulation. J Cereb Blood Flow Metab 31:832-841.

Shmuel A, Yacoub E, Pfeuffer J, Van de Moortele PF, Adriany G, Hu X, 
Ugurbil K (2002) Sustained negative BOLD, blood flow and oxygen consumption response and its coupling to the positive response in the human brain. Neuron 36:1195-1210.

Shmuel A, Augath M, Oeltermann A, Logothetis NK (2006) Negative functional MRI response correlates with decreases in neuronal activity in monkey visual area V1. Nat Neurosci 9:569-577.

Slaght SJ, Paz T, Chavez M, Deniau JM, Mahon S, Charpier S (2004) On the activity of the corticostriatal networks during spike-and-wave discharges in a genetic model of absence epilepsy. J Neurosci 24:6816-6825.

Smith AJ, Blumenfeld H, Behar KL, Rothman DL, Shulman RG, Hyder F (2002) Cerebral energetics and spiking frequency: the neurophysiological basis of fMRI. Proc Natl Acad Sci U S A 99:10765-10770.

Smith AM, Lewis BK, Ruttimann UE, Ye FQ, Sinnwell TM, Yang Y, Duyn JH, Frank JA (1999) Investigation of low frequency drift in fMRI signal. Neuroimage 9:526-533.

Stefanovic B, Warnking JM, Pike GB (2004) Hemodynamic and metabolic responses to neuronal inhibition. Neuroimage 22:771-778.

Steriade M, Jones EG, McCormick DA, eds (1997) Thalamus. Amsterdam: Elsevier Science.
Suh M, Bahar S, Mehta AD, Schwartz TH (2005) Temporal dependence in uncoupling of blood volume and oxygenation during interictal epileptiform events in rat neocortex. J Neurosci 25:68-77.

Suh M, Ma H, Zhao M, Sharif S, Schwartz TH (2006) Neurovascular coupling and oximetry during epileptic events. Mol Neurobiol 33: 181-197.

Tenney JR, Duong TQ, King JA, Ludwig R, Ferris CF (2003) Corticothalamic modulation during absence seizures in rats: a functional MRI assessment. Epilepsia 44:1133-1140.

Tenney JR, Duong TQ, King JA, Ferris CF (2004) FMRI of brain activation in a genetic rat model of absence seizures. Epilepsia 45:576582.

Vaudano AE, Laufs H, Kiebel SJ, Carmichael DW, Hamandi K, Guye M, Thornton R, Rodionov R, Friston KJ, Duncan JS, Lemieux L (2009) Causal hierarchy within the thalamo-cortical network in spike and wave discharges. PLoS One 4:e6475.

Zhao M, Ma H, Suh M, Schwartz TH (2009) Spatiotemporal dynamics of perfusion and oximetry during ictal discharges in the rat neocortex. J Neurosci 29:2814-2823. 